\title{
VESTIBULAR AUTONOMIC REGULATION: OVERVIEW AND CONCLUSIONS OF A RECENT WORKSHOP AT THE UNIVERSITY OF PITTSBURGH
}

\section{Introduction}

A key role of the central nervous system is to provide for homeostasis, or a stable internal milieu. One of the most profound challenges to homeostasis occurs when a human or other animal moves or changes posture. In particular, some movements, such as standing in humans or nose-up body pitch in quadrupeds, can threaten the maintenance of stable blood pressure and blood oxygenation. Unless compensation takes place quickly, these movements produce 1) blood pooling in the lower body that results in orthostatic hypotension and 2) a change in the resting length of the respiratory muscles that results in decreased air flow through the lungs (see papers in this journal issue for further explanation). Many body sensors, including arterial baroreceptors, receptors in the heart, receptors in limb veins, receptors in the lungs, stretch receptors in respiratory muscles, and central and peripheral chemoreceptors, detect disturbances in homeostasis and trigger appropriate compensatory responses. However, effective maintenance of homeostasis would seem to require that compensation for the effects of movement on circulation and respiration begin even before the internal environment has been affected. One mechanism for accomplishing this would be through the actions of the vestibular system, which detects head position and head movements, and could thus provide "feed-forward" information to the brainstem autonomic centers, resulting in corrections in blood pressure and ventilation during changes in body position.

The National Institute on Deafness and Other Communication Disorders (NIDCD) of the Na- tional Institutes of Health (NIH) joined the Life Sciences Division of the National Aeronautics and Space Administration (NASA) in co-sponsoring a two-day workshop to consider the role of the vestibular system in regulating cardiovascular function and respiratory muscle activity on Earth and in the microgravity environment of space. A second purpose of this workshop was to explore the role of the vestibular system in producing autonomic disturbances associated with terrestrial and space motion sickness and with balance disorders. This workshop was held at the University of Pittsburgh on April 23 and 24, 1996, and included presentations by invited speakers as well as roundtable discussion sessions to provide direction for future research efforts. The University of Pittsburgh was chosen as the venue for this meeting because of the leadership of scientists at this institution in research on vestibular-autonomic regulation. The co-sponsorship of this workshop by NASA's Life Sciences Division and the NIDCD is one example of the many productive scientific collaborations between these two agencies to address their mutual research interests. The study of interactions between the vestibular system and the autonomic nervous system is important in the research missions of both agencies.

Approximately 75 basic and clinical scientists with research interests in the vestibular system, the autonomic nervous system, the cardiovascular system, emesis, and the space life sciences attended the workshop. This meeting was divided into four sessions, which considered 1) the importance of the vestibular system in autonomic regulation, 2) possible links between effects of microgravity on the vestibular 
and cardiovascular systems, 3) new strategies for treatment and prevention of terrestrial and space motion sickness, and 4) future research needs in the area of vestibular autonomic regulation. The workshop also featured a presentation regarding the physiological effects of space flight by a former astronaut, Dr. F. Andrew Gaffney, a cardiologist from Vanderbilt University who flew on the Shuttle Columbia in 1991 (mission STS-40).

The scientific highlights of this workshop are summarized below. The papers in this journal issue expand upon many of the issues raised at the Workshop on Vestibular Autonomic Regulation.

\section{Evidence for Vestibular Autonomic Regulation}

It has long been known that a functioning vestibular system is essential for producing motion sickness, suggesting that inputs from the vestibular labyrinth are coupled to the brainstem autonomic centers. However, the extent of the vestibular autonomic connections was not realized until recently, when neuroanatomical and neurophysiological experiments demonstrated vestibular inputs to brainstem centers that are involved in control of the sympathetic nervous system and the respiratory system. Furthermore, removal of vestibular inputs to the brainstem compromises the ability to adjust blood pressure during unexpected changes in posture. Vestibular-autonomic influences appear to be multifaceted, in that vestibular inputs are integrated with other signals at many stages. The circuitry that mediates vestibulo-autonomic responses appears to be influenced by several brain regions, including the cerebral cortex. For example, motion sickness is not as severe in experienced astronauts who have flown in space before and have learned to predict the unusual spatial cues that occur in microgravity. The cerebellum also provides inputs to the brainstem regions that integrate vestibular and visceral inputs. However, the precise role of the cerebellum and cerebral cortex in influencing vestibular autonomic regulation is yet to be determined.

Recent evidence also suggests that the vestibular system has a number of roles in addition to the production of postural adjustments, head stabilization, and compensatory eye movements during changes in head position. For example, the vestibular system may functionally interact with pontine sleep control centers. Such actions of the vestibular system have been explored only in a rudimentary fashion, and merit further study.

\section{Microgravity-related Changes in Vestibular Autonomic Regulation}

Autonomic problems accompany exposure to unusual gravitational environments, providing more evidence for a vestibular-autonomic link. In the space flight environment, the otolith organs are no longer exposed to net gravitational forces. Thus, the otolith organs are not stimulated by tilting the head, but rather are stimulated only by linear acceleration of the head. This alteration in otolith function causes both anatomical and physiological changes in the vestibular system. Anatomic studies of the synaptic connections of the otolithic hair cells of rats after space flights of 9 and 14 days have revealed that a loss of gravitational input causes an increase in synaptic innervation, especially in the Type II hair cells. Exposure to increased gravitational forces (hypergravity) during centrifugation causes a decrease in synaptic innervation of Type II hair cells. Other plastic changes are known to occur in vestibular connections in the central nervous system during exposure to microgravity. These studies, which clearly show the ability of the vestibular system to adapt rapidly to an altered gravitational environment, have important implications for longduration space flight.

A number of autonomic disturbances may result from the alterations in otolith organ stimulation during space flight. For example, space motion sickness has been linked to altered otolithic function in microgravity. This condition can compromise crew performance, and even incapacitate a crew member for several days at the beginning of space flight. Moreover, crew members may experience "Earth sickness" upon return to a gravitational environment, which can impair performance during the landing of the spacecraft. Although some medica- 
tions provide relief for some symptoms of space motion sickness, there is to date no cure for this malady, and the underlying mechanisms are not well understood.

Microgravity-related effects on the otolith organs also may be partly responsible for postflight orthostatic hypotension. Intolerance to orthostatic stress after return to Earth was one of the first recognized physiologic effects of space flight and has been observed from the earliest flights of the Mercury and Gemini astronauts to the present day. The severity of this orthostatic intolerance may or may not be greater after long-duration space flight, but the length of time that the intolerance persists after return to Earth appears to be correlated with the duration of microgravity exposure.

A number of physical changes occur in space that can affect the cardiovascular system and contribute to postflight orthostatic hypotension. Loss of the hydrostatic gradient causes an almost immediate redistribution of fluids in the body from the legs and lower body toward the central circulation and the head. Facial puffiness and venous engorgement can be seen readily, and astronauts experience nasal stuffiness and often headaches; these effects are long lasting. Cerebral pressures may be affected, but have not been measured to date. Increased fluids in the central circulation are reflected in greater cardiac volumes and greater stroke volumes; these effects can produce changes in autonomic reflexes and hormone levels that result in a transient decrease in total body water and a persistent decrease in plasma volume. The decrease in plasma volume is one factor that contributes to a loss of tolerance to orthostatic stress in astronauts returning to a gravitational environment after space flight. Another factor contributing to this orthostatic intolerance is a decreased responsiveness of the high-pressure arterial baroreceptor reflex, as expressed in changes in the heart rate response to stimulation of carotid baroreceptors. These mechanisms, however, do not account for the total magnitude of the postflight orthostatic intolerance. Because the otolith organs participate in regulating blood pressure, it is possible that the plastic changes in the otolith organs and in the central processing of otolith signals resulting from exposure to micro- gravity can contribute to the orthostatic hypotension following return to Earth. Thus, a better understanding of the mechanisms of orthostatic intolerance, including the possible contribution of vestibulo-autonomic interactions, is needed.

\section{Implications of Vestibular Autonomic Regulation}

The fact that vestibular stimulation has autonomic effects is of relevance to all vestibular researchers. Autonomic effects of vestibular stimulation, or changes in autonomic functioning resulting from vestibular lesions, can indirectly have effects on other vestibular reflexes. For example, fluid loss associated with vestibularinduced emesis or orthostatic hypotension resulting from vestibular lesions could result in lightheadedness during rapid and unexpected changes in posture. This lightheadedness could indirectly have effects on vestibulospinal and vestibulo-ocular reflexes. Furthermore, motion sickness and dysfunction in vestibular autonomic regulation can result in distress and reduced attention to environmental stimuli and thereby alter other vestibular reflexes.

Connections between the vestibular system and brainstem autonomic centers can also be important in clinical medicine. As discussed above, vestibular lesions can increase the susceptibility for orthostatic hypotension and may decrease the ability to rapidly adjust respiration during movement. Neuroanatomical studies showing direct connections between the vestibular nuclei, the locus coeruleus, and brainstem pathways that process visceral sensory information also provide a potential neural substrate for the autonomic and affective signs and symptoms often associated with vestibular dysfunction. Clinical studies have shown a close linkage between generalized anxiety, panic disorder, agoraphobia, and vestibular dysfunction. Common to patients with panic disorder and agoraphobia are heightened sensations of discomfort with motion and with changes in body position in space. Thus, alterations in vestibular functioning may contribute to some psychiatric disorders.

Vestibular autonomic regulation also has important implications for the space life sciences. 
Substantial data already indicate that vestibular-autonomic pathways are at least partially responsible for space motion sickness, and, as discussed above, plastic changes in the vestibular system during space flight also may be partially responsible for postflight orthostatic intolerance. Other physiological problems experienced by astronauts, including sleep disturbances, could additionally be linked with microgravity-related changes in the vestibular system. Further research is required to determine the relationship between changes in vestibular functioning and alterations in a number of physiological processes during and subsequent to spaceflight.

With the longer duration space flights planned for the future, including the International Space Station assignments of 90 to 180 days and missions to Mars, which may require 3 years, vestibular-autonomic disturbances may become of even greater significance to NASA. A Mars mission will be further complicated by the requirement for crew members to egress in the partial-gravity environment of Mars without assistance after a flight of many months. Thus, it is imperative that we understand better the implications of changes in vestibular autonomic regulation during space flight and that we develop countermeasures to prevent or compensate for these changes.

\section{Areas for Future Research}

The workshop determined several areas in which research is needed. The role of functionally unloading the otolith organs in space in producing a number of physiological disturbances, including postflight orthostatic intolerance, postflight changes in respiratory muscle activation during movement and changes in posture, changes in sleep patterns, and so on, should be studied. This research is particularly germane at the present time, because space flights that will last from months to years are currently being planned. Before such long-term missions will be routinely possible, countermeasures must be designed for the deleterious effects that months of exposure to microgravity will have on the body.

It will also be important to determine both the physiological properties and the pharmaco- logical properties of the neural circuitry responsible for nausea and emesis, including those neural pathways involved in producing motion sickness. Such research will allow for the development of better anti-emetic agents; the current anti-emetic drugs mainly treat the "symptoms" of motion sickness, and do not affect the mechanisms that produce this condition. Animal research will be required to achieve this goal.

It is also well established that some brainstem monoaminergic neurons, including those that synthesize norepinephrine and serotonin, are influenced by the vestibular system. These monoaminergic neurons have influences on many brain systems, and dysfunction in monoaminergic neurotransmission has been linked to a number of psychiatric disturbances. The implications of vestibular inputs to the monoaminergic neurons, and the relationship of vestibular dysfunction to psychiatric diseases, must be explored in detail.

Preliminary evidence suggests that the vestibular system has influences on the pontine neurons that are involved in regulating sleep cycles. These influences should be examined in more detail. In addition, vestibular stimulation has been shown to affect the activity of respiratory muscles. The importance of vestibular inputs in the regulation of breathing and blood oxygenation should be explored. Recent evidence suggests that rocking, which stimulates the vestibular system, tends to stabilize the respiration pattern in infants with sudden infant death syndrome (SIDS). Thus, possible links between the vestibular system and diseases that affect the respiratory system should also be explored.

\section{Organization of this Journal Issue}

The papers in this issue of the Journal of Vestibular Research were contributed by participants in the Workshop on Vestibular Autonomic Regulation. The issue will begin with reviews providing neuroanatomical, neurophysiological, and clinical evidence to suggest that the vestibular system participates in autonomic regulation. These reviews will be followed by papers suggesting the role of the vestibular system in cardiovascular control under several con- 
ditions, including physical exercise. Another group of manuscripts will discuss space and terrestrial motion sickness and potential new treatments for these conditions. The next group of papers will discuss newly discovered roles of the vestibular system, including the possible participation in the regulation of sleep-wake cycles. The final papers in this journal issue are original research reports providing new information on the role of the vestibular system in autonomic control.
B. J. Yates

D. A. Sklare

M. A. B. Frey 